\title{
25 Research Soure \\ Biodata Mining-based Elucidation of Mechanisms of Hesperetin as a Candidate for Atherosclerosis
}

\section{Lianzhou Huang \\ Jinan University \\ Zexiu Huang \\ Jinan University \\ Yuanqiu Chen \\ Jinan University \\ Xin Jin \\ Jinan University \\ Ji Xiao \\ Jinan University \\ Zhe Ren \\ Jinan University}

Yiliang Wang ( $\nabla$ wang_yiliang@sina.cn )

College of Life science and Technology, Guangzhou Jinan Biomedicine Research and Development Center, Jinan University, Guangzhou 510632, PR China; https://orcid.org/0000-0002-0650-5662

\section{Research}

Keywords: Hesperetin, anti-inflammatory effect, Citrus $\times$ aurantium L., pharmacological mechanisms

Posted Date: September 30th, 2020

DOI: https://doi.org/10.21203/rs.3.rs-83163/v1

License: (c) (i) This work is licensed under a Creative Commons Attribution 4.0 International License.

Read Full License 


\section{Abstract}

\section{Background}

Hesperetin, an active ingredient derived from Citrus $\times$ aurantium L., possesses a wide range of biological activities, including anti-inflammatory, anti-oxidation, and anti-cancer activity. Notably, hesperetin has been proposed as a candidate for atherosclerosis owing to the lipid-regulating and anti-inflammatory effect, while the underlying mechanisms remains obscure.

\section{Results}

In our present study, the pharmacological and molecular properties of hesperetin were first evaluated to determine the druggability of hesperetin. Subsequently, 53 hesperetin-atherosclerosis crossover targets were collected to establish the protein-protein interaction network. The result of Gene Ontology enrichment analysis indicated that the crossover targets were involved in the regulation of lipid metabolism and inflammatory response. Moreover, the Kyoto Encyclopedia of Genes and Genomes pathway analyses demonstrated that the crossover targets were highly correlated with the pathogenesis of atherosclerosis, such as fluid shear stress and atherosclerosis pathway and the TNF signaling pathway. Finally, an entire hesperetin-target-pathway network was constructed to provide a systematic overview of the pharmacological mechanisms of action of hesperetin against atherosclerosis.

\section{Conclusions}

The pharmacological mechanisms of actions of hesperetin against atherosclerosis was unveiled based on biodata mining from the public database and the bioinformatics data analysis-based strategy in this study, contributing to a deeper understanding of the molecular mechanisms of hesperetin in the treatment of atherosclerosis. Based on the results of network pharmacology analysis, we can conclude that hesperetin is surely an excellent candidate for atherosclerosis. We believe our work would be beneficial for further research and development of hesperetin as a natural active ingredient derived from Citrus $\times$ aurantium $L$. for the treatment of atherosclerosis.

\section{Introduction}

Atherosclerosis is a chronic, progressive, inflammatory disease characterized by excessive accumulation of lipids in the walls of large arteries (1). Although there are multiple theories to describe the occurrence and development of atherosclerosis, the pathogenesis of atherosclerosis has not been fully recognized (2). Nevertheless, the inflammatory activation induced by abnormal lipid accumulation in the walls of large arteries plays a central role in the pathological process of atherosclerosis (3). In particular, lowdensity lipoprotein $(L D L)$ is considered to be the main contributor to the formation of atherosclerotic plaque, excessive accumulation of which in the inner walls of the arteries could be oxidized to form oxidized LDL (4). Owing to the inflammatory activation, endothelial cells produce numerous inflammatory cytokines, such as chemoattractant protein-1 (MCP-1), vascular cell adhesion molecule 1 (VCAM-1), E- 
selectin, and P-selectin, which recruit monocytes and leukocytes to infiltrate at inflammatory sites (5). Afterward, monocytes will differentiation into macrophages and transform into foam cells eventually via the internalization of oxidized LDL, which constitute the core of the atherosclerotic plaque (6). Therefore, the clinical treatment of atherosclerosis mainly focuses on interfering with the biosynthesis of lipids in the organism. The first-line anti-atherosclerosis drugs mainly include 3-hydroxy-3-methyl glutaryl coenzymes A (HMG-CoA) reductase inhibitors, such as lovastatin, simvastatin, and pravastatin, which can substantially block the biosynthesis of lipids via inactivating the HMG-CoA reductase (7). However, stains could cause serious damage to the liver (8). Moreover, 31 patients died of severe rhabdomyolysis due to the use of cerivastatin (9). Thus, the research and development of toxic-less and effective-more anti-atherosclerosis drugs are urgently needed.

The traditional medicinal plant plays a critical role in disease prevention and treatment, the active ingredients from which are also a tremendous source of lead compound (10). For instance, hesperetin, a bioflavonoid derived from the Citrus $\times$ aurantium L. (11), has been verified to exhibit a variety of biological activities, such as anti-hypertension (12), anti-oxidation (13), anti-diabetes (14), anti-cancer (15), neuroprotection (16), and attenuation of renal interstitial fibrosis (17). Moreover, hesperetin has been proposed for the treatment of atherosclerosis attributing to the lipid-regulating and anti-inflammatory effects (18). However, the underlying pharmacological mechanism remains to be systematically explored. The concept "network pharmacology" was proposed by Hopkins in 2007 (19). Based on the principle of systems biology and network analysis, network pharmacology-based strategy was adopted to recognize the pharmacological mechanism of a drug in the interaction networks of "drug-disease-target-pathway" (20). Combined systems biology with computer virtual technology, network pharmacology displays great superiority for studying the pharmacological mechanism of multiple ingredients in the traditional medicinal plant in a high-throughput manner (21).

Accordingly, this study aimed to explore the pharmacological mechanism of hesperetin for the treatment of atherosclerosis based on biodata mining derived from the public database. Our research successfully explained the molecular mechanism of actions of hesperetin for the treatment of atherosclerosis based on network pharmacology. We believe our findings would be beneficial for a comprehensive understanding of the mechanism of actions of hesperetin against atherosclerosis. Moreover, we believe our study would promote the study of the pharmacological mechanism of action of active ingredients derived from the traditional medicinal plant in the future.

\section{Materials And Methods}

\section{Assessment of pharmacological properties of hesperetin}

Traditional Chinese medicine systems pharmacy database and analysis platform (TCMSP, https://tcmspw.com/tcmsp.php version: 2.3) provided detailed pharmacological and molecular properties data of hesperetin, including 12 parameters associated with the absorption, distribution, metabolism, and excretion (ADME) of hesperetin in the organism (22). Among them, oral bioavailability (OB) and drug 
likeness (DL) acts as a critical role in the absorption of orally administered hesperetin in the gastrointestinal tract, which is commonly served as the basic criteria in evaluating the druggability of hesperetin. Therefore, the chemical name "hesperetin" was entered into the search box of the TCMSP database. Meanwhile, " $\mathrm{DL} \geq 0.18$ and $\mathrm{OB} \geq 30 \%$ " was regarded as the criteria to assess the druggability of hesperetin in this study. Of note, the Mol2 file containing the 3-dimensional structure of hesperetin can also be gained in the TCMSP database to predict the potential targets of hesperetin.

\section{Targets prediction of hesperetin}

The PharmMapper database is a freely accessed web-server to identify potential targets of a compound via a reverse pharmacophore mapping approach (http://www.lilab-ecust.cn/pharmmapper/ version: 2017) (23). Following the Mol2 file of hesperetin submitted to the PharmMapper database, the top 300 protein targets of hesperetin from Homo Sapiens will be obtained sorted by fit score in descending order. Of note, the protein name of these targets could be converted to the corresponding gene names by utilizing the "Retrieve/ID mapping" tool in the UniProt database to standardize the targets of hesperetin (https://www.uniprot.org/ last modified date: October 15, 2019).

\section{Targets identification of atherosclerosis}

Targets associated with atherosclerosis were derived from the GeneCards database (https://www.genecards.org/ version 4.14), which is an integrative database enabling us to navigate the targets associated with atherosclerosis from Homo Sapiens (24). In this study, we retrieved the targets associated with atherosclerosis from the GeneCards database by entering "atherosclerosis" as a keyword in the search box.

\section{Screening of the hesperetin-atherosclerosis crossover targets and construction of PPI network}

Venn diagram in the Bioinformatics website was employed to screening out the hesperetinatherosclerosis crossover targets (25)

(http://www.bioinformatics.com.cn/static/others/jvenn/example.html).Subsequently, the GeneMANIA website (https://genemania.org/ version: 3.6.0) was utilized to characterize the protein-protein interactions (PPIs) between the hesperetin-atherosclerosis crossover targets (26). Inputting the hesperetin-atherosclerosis crossover targets into the search box, the GeneMANIA website would represent the PPI network illustrating the possible interactions of these crossover targets, including co-expression, co-localization, physical and genetic interaction, shared protein domains, pathway and predicted interaction.

\section{KEGG and GO enrichment analysis}

Kyoto Encyclopedia of genes and genes (KEGG) and Gene Ontology (GO) enrichment analysis were then performed in the Metascape website to analysis the corresponding cell signaling pathways, biological process (BP), molecular function (MF), and cellular component (CC) related to the hesperetinatherosclerosis crossover targets (https://metascape.org/gp/index.html\#/main/step1 last update date: 
March 20, 2020) (27). Giving a require in the search box in the Metascape website, the involved cell signaling pathways, biological process, molecular function, and cellular component related to the hesperetin-atherosclerosis crossover targets will be enriched. Of note, $\mathrm{P}<0.01$ was regarded as an obvious discrepancy during enrichment analysis.

\section{Construction of the hesperetin-target-pathway network}

To understand the intricate connections between hesperetin, targets, and pathways, the "hesperetintarget-pathway" network was visualized by Cytoscape software (Cytoscape version: 3.7.2) (28).

\section{Results}

\section{Chemical structure and pharmacological data of hesperetin.}

ADME describes the distribution of hesperetin inside the human body, which is needed to be considered while assessing the therapeutic efficacy of hesperetin against atherosclerosis (29). TCMSP database offered ADME-related 12 pharmacokinetic parameters, including molecular weight (MW), logarithm of the octanol-water partition coefficient (AlogP), hydrogen-bond donor (Hdon), hydrogen-bond acceptor (Hacc), oral bioavailability (OB\%), Caco-2 permeability (Caco-2), blood brain barrier permeability (BBB), drug likeness (DL), fractional negative surface area (FASA), topological polar surface area (TPSA), rotatable bonds (RBN), and drug half-life $(\mathrm{HL})$. The pharmacological properties of hesperetin have been investigated by referring to the TCMSP database (Table 1). Of note, the OB and DL, two crucial parameters for evaluating the druggability of hesperetin, were higher than the threshold value (" $D L \geq 0.18$ and $\mathrm{OB} \geq 30 \%$ "), suggesting that hesperetin is a druggable candidate.

\section{Identification of hesperetin-atherosclerosis crossover targets}

As described in the "Materials and methods" section, the 294 targets of hesperetin from Homo Sapiens were acquired from the PharmMapper database after removing the duplicates and non-human targets. Besides, 4346 targets associated with atherosclerosis were collected from the GeneCards database while a total of 383 targets were used for further investigation by filtering out low correlative targets (relevance score $<6$ ). Moreover, 53 hesperetin-atherosclerosis crossover targets were identified by the Venn diagram in the Bioinformatics website (Figure 2A). The gene names and gene symbols of the crossover targets have been listed (Table 2).

\section{PPI network establishment}

To explore the functional connections among these crossover targets, we constructed the PPI network of the crossover targets through employing the GeneMANIA database. Among the 53 targets and their interacting 20 proteins, $49.42 \%$ showed the co-expression characteristics, $10.05 \%$ had physical connections, $7.04 \%$ showed the characteristics of co-localization, and other connections, such as shared proteins, predicted interactions and genetic interactions have been quantified (Figure 3A). Furthermore, the crossover targets were subsequently sorted by "Degree value" in descending order residents in 
Cytoscape software (Table 3). Of note, as indicated by their degree value calculated by Cytoscape, Albumin, differing from HMG-CoA reductase, could be dug out for the further development of antiatherosclerosis drugs.

\section{GO and KEGG enrichment analysis}

To systematically decipher the mode of action of hesperetin against atherosclerosis, GO and KEGG pathway enrichment analysis were carried out to identify the molecular functions, biological processes, cellular components, and cellular signaling pathways of these crossover targets. The KEGG pathway enrichment analysis suggested that the crossover targets are closely associated with the fluid shear stress and atherosclerosis pathway (hsa05418), AGE-RAGE signaling pathway in diabetic complications pathway (hsa04933), TNF signaling pathway (hsa04668), and insulin resistance pathway (hsa04931) in the top five pathways (Figure 4A). GO annotation revealed that the crossover targets were involved in lipid metabolism and inflammatory response, such as regulation of inflammatory response (G0:0006869) and lipid transport (GO:0050727) were included. Collectively, these crossover targets were highly associated with the pathogenesis of atherosclerosis.

\section{Construction of the hesperetin-targets-pathway network}

Based on the crossover targets and the corresponding pathways, an entire "hesperetin-targets-pathway" network was constructed using Cytoscape software. In general, this "hesperetin-targets-pathway" network has one compound and 53 targets, involving 58 cell signaling pathways, 112 nodes, and 412 edges. As presented in this network, the targets of hesperetin and its corresponding pathway were connected to obtain a visual view. For instance, hesperetin could regulate the fluid shear stress and atherosclerosis pathway (hsa05418) by interacting with its target including AKT2, RHOA, MAPK14, GSTM1, KDR, MMP2, MMP9, NOS3, MAPK8, SELE, and SRC, thereby ameliorating development and progression of atherosclerosis (Figure $5 \mathrm{~A}$ ).

\section{Discussion}

Hesperetin exhibited excellent lipid-regulating and anti-inflammatory activity, which was deemed as a promising candidate against atherosclerosis (30). However, the mechanism of actions of hesperetin against atherosclerosis remains obscure. Therefore, a bioinformatics data analysis-based strategy was applied in this study to investigate the pharmacological mechanism of hesperetin for the treatment of atherosclerosis. Specifically, the pharmacological properties of hesperetin meet the threshold requirements, implying the excellent druggability of hesperetin for anti-atherosclerosis drug development. Of note, 294 potential targets of hesperetin and 383 targets related to atherosclerosis were identified. Afterward, 53 crossover targets were obtained for further study, in which targets closely coincide with the pathogenesis of atherosclerosis were observed, such as APOA2, SELP, PPARG, SELP. The PPI network provided information on interactions between these crossover targets and 20 genes associated with the crossover targets. The subsequent GO and KEGG enrichment analysis indicated that the crossover targets were closely related to the lipid metabolism and activation of inflammation such as fluid shear stress and 
atherosclerosis pathway (hsa05418), regulation of inflammatory response (G0:0006869) and lipid transport (G0:0050727). Of note, GO and KEGG pathway enrichment analysis was also consistent with the previous report that hesperetin for the treatment of atherosclerosis depended on its lipid-regulating and anti-inflammatory activity. Finally, the hesperetin-target-pathway network was constructed to depict a visual view of the mechanism of actions of hesperetin for the treatment of atherosclerosis.

Notably, differing from HMG-CoA reductase, we identified another potential target "Albumin", whereas experimental verification is needed for determining the possibility of Albumin as a novel target for antiatherosclerosis drug development. During KEGG enrichment analysis, the high correlation between crossover targets and cancer leading us to hypothesize that there might be some unknown connections between atherosclerosis and cancer, which remains to be explored in larger studies. Of note, we also admitted that the elucidation of the pharmacological mechanism of hesperetin against atherosclerosis by data mining and the bioinformatics-based approach was a prediction, and the more precise mechanism remains to be verified by large-scale experiments.

In summary, it was the first time that the data mining from the public database and the bioinformatics data analysis-based strategy was proposed to uncover the pharmacological mechanism of actions of hesperetin in alleviating atherosclerosis. Based on the results of network pharmacology analysis, hesperetin may be a superior candidate for the development of a multi-target and multi-pathway antiatherosclerosis medicament. We believe our findings would contribute to the further research and development of hesperetin as a natural active ingredient derived from traditional medicinal plants for the treatment of atherosclerosis.

\section{Abbreviations}

LDL, low-density lipoprotein; MCP-1, chemoattractant protein-1; VCAM-1, vascular cell adhesion molecule 1; HMG-CoA, 3-hydroxy-3-methyl glutaryl coenzyme A; TCM, traditional Chinese medicine; TCMSP, Traditional Chinese medicine systems pharmacy database and analysis platform; A, absorption, D, distribution; $\mathrm{M}$, metabolism; $\mathrm{E}$, excretion; $\mathrm{OB}$ oral bioavailability; $\mathrm{DL}$, drug likeness; PPIs, intersecting protein-protein interactions; KEGG, Kyoto Encyclopedia of genes and genes; GO, Gene Ontology; BP, biological process; MF, molecular function; CC, cellular component; MW, molecular weight; AlogP, logarithm of the octanol-water partition coefficient; Hdon, hydrogen-bond donor; Hacc, hydrogen-bond acceptor; Caco-2, Caco-2 permeability; BBB, blood brain barrier permeability; FASA, fractional negative surface area; TPSA, topological polar surface area;RBN, rotatable bonds; $\mathrm{HL}$, drug half-life.

\section{Declarations}

\section{Acknowledgments}

We would like to acknowledge all participants in this study. We thank you for your collection and analysis of the data presented in this study. Besides, we appreciate your critical comments for improving the 
quality of this manuscript.

\section{Funding}

This research did not receive any specific grant from funding agencies.

\section{Availability of data and materials}

All data generated or analyzed presented in this study are available in the database listed in this article.

\section{Author contributions}

Lianzhou Huang was responsible for data retrieval, data analysis, and editing of the manuscript. Yiliang Wang is in charge of the critical review of the manuscript. All authors agreed to the publication of this manuscript.

\section{Ethics approval and consent to participate}

Not applicable.

\section{Consent for publication}

Not applicable.

\section{Competing interests}

All author declares no potential conflict of interest.

\section{References}

1. Chanet A, Milenkovic D, Claude S, Maier JA, Khan MK, Rakotomanomana N, et al. Flavanone metabolites decrease monocyte adhesion to TNF-a-activated endothelial cells by modulating expression of atherosclerosis-related genes. British journal of nutrition. 2013;110(4):587-98.

2. Libby P, Ridker PM, Hansson GK. Progress and challenges in translating the biology of atherosclerosis. Nature. 2011;473(7347):317-25.

3. Fatkhullina AR, Peshkova IO, Koltsova EK. The Role of Cytokines in the Development of Atherosclerosis. Biochemistry Biokhimiia. 2016;81(11):1358-70.

4. Kattoor AJ, Kanuri SH, Mehta JL. Role of Ox-LDL and LOX-1 in Atherogenesis. Current medicinal chemistry. 2019;26(9):1693-700.

5. Geovanini GR, Libby P. Atherosclerosis and inflammation: overview and updates. Clinical science (London, England : 1979). 2018;132(12):1243-52.

6. Groh L, Keating ST, Joosten LAB, Netea MG, Riksen NP. Monocyte and macrophage immunometabolism in atherosclerosis. Seminars in immunopathology. 2018;40(2):203-14. 
7. Vaughan CJ, Gotto AM, Jr., Basson CT. The evolving role of statins in the management of atherosclerosis. Journal of the American College of Cardiology. 2000;35(1):1-10.

8. Vargas JI, Arrese M, Shah VH, Arab JP. Use of Statins in Patients with Chronic Liver Disease and Cirrhosis: Current Views and Prospects. Current gastroenterology reports. 2017;19(9):43.

9. Tobert JA. Lovastatin and beyond: the history of the HMG-CoA reductase inhibitors. Nature Reviews Drug Discovery. 2003;2(7):517-26.

10. Rahman IU, Afzal A, Iqbal Z, ljaz F, Ali N, Shah M, et al. Historical perspectives of ethnobotany. Clinics in dermatology. 2019;37(4):382-8.

11. Wang $\mathrm{H}$, Wang $\mathrm{H}-\mathrm{f}$, Zhang $\mathrm{H}$, Wang $\mathrm{C}$, Chen $\mathrm{Y}-\mathrm{f}, \mathrm{Ma} \mathrm{R}$, et al. Inhibitory effects of hesperetin on Nav1.5 channels stably expressed in HEK 293 cells and on the voltage-gated cardiac sodium current in human atrial myocytes. Acta Pharmacologica Sinica. 2016;37(12):1563-73.

12. Yamamoto M, Suzuki A, Hase T. Short-term effects of glucosyl hesperidin and hesperetin on blood pressure and vascular endothelial function in spontaneously hypertensive rats. Journal of nutritional science and vitaminology. 2008;54(1):95-8.

13. Kim HJ, Jeon SM, Lee MK, Cho YY, Kwon EY, Lee JH, et al. Comparison of hesperetin and its metabolites for cholesterol-lowering and antioxidative efficacy in hypercholesterolemic hamsters. Journal of medicinal food. 2010;13(4):808-14.

14. Sharma M, Akhtar N, Sambhav K, Shete G, Bansal AK, Sharma SS. Emerging potential of citrus flavanones as an antioxidant in diabetes and its complications. Current topics in medicinal chemistry. 2015;15(2):187-95.

15. Roohbakhsh A, Parhiz H, Soltani F, Rezaee R, Iranshahi M. Molecular mechanisms behind the biological effects of hesperidin and hesperetin for the prevention of cancer and cardiovascular diseases. Life sciences. 2015;124:64-74.

16. Kheradmand E, Hajizadeh Moghaddam A, Zare M. Neuroprotective effect of hesperetin and nanohesperetin on recognition memory impairment and the elevated oxygen stress in rat model of Alzheimer's disease. Biomedicine \& pharmacotherapy = Biomedecine \& pharmacotherapie. 2018;97:1096-101.

17. Wang HW, Shi L, Xu YP, Qin XY, Wang QZ. Hesperetin alleviates renal interstitial fibrosis by inhibiting tubular epithelial-mesenchymal transition in vivo and in vitro. Experimental and therapeutic medicine. 2017;14(4):3713-9.

18. Sugasawa N, Katagi A, Kurobe H, Nakayama T, Nishio C, Takumi H, et al. Inhibition of Atherosclerotic Plaque Development by Oral Administration of a-Glucosyl Hesperidin and Water-Dispersible Hesperetin in Apolipoprotein E Knockout Mice. Journal of the American College of Nutrition. 2019;38(1):15-22.

19. Hopkins AL. Network pharmacology. Nature Biotechnology. 2007;25(10):1110-1.

20. Hopkins AL. Network pharmacology: the next paradigm in drug discovery. Nature Chemical Biology. 2008;4(11):682-90. 
21. Boezio B, Audouze K, Ducrot P, Taboureau O. Network-based Approaches in Pharmacology. Molecular informatics. 2017;36(10).

22. Ru J, Li P, Wang J, Zhou W, Li B, Huang C, et al. TCMSP: a database of systems pharmacology for drug discovery from herbal medicines. Journal of cheminformatics. 2014;6:13.

23. Liu X, Ouyang S, Yu B, Liu Y, Huang K, Gong J, et al. PharmMapper server: a web server for potential drug target identification using pharmacophore mapping approach. Nucleic Acids Research. 2010;38(suppl_2):W609-W14.

24. Stelzer G, Rosen N, Plaschkes I, Zimmerman S, Twik M, Fishilevich S, et al. The GeneCards Suite: From Gene Data Mining to Disease Genome Sequence Analyses. Current Protocols in Bioinformatics. 2016;54(1):1.30.1-1..3.

25. Bardou P, Mariette J, Escudié F, Djemiel C, Klopp C. jvenn: an interactive Venn diagram viewer. BMC Bioinformatics. 2014;15(1):293.

26. Warde-Farley D, Donaldson SL, Comes O, Zuberi K, Badrawi R, Chao P, et al. The GeneMANIA prediction server: biological network integration for gene prioritization and predicting gene function. Nucleic Acids Res. 2010;38(Web Server issue):W214-20.

27. Zhou Y, Zhou B, Pache L, Chang M. Metascape provides a biologist-oriented resource for the analysis of systems-level datasets. 2019;10(1):1523.

28. Shannon P, Markiel A, Ozier O, Baliga NS, Wang JT, Ramage D, et al. Cytoscape: a software environment for integrated models of biomolecular interaction networks. Genome research. 2003;13(11):2498-504.

29. Tibbitts J, Canter D, Graff R, Smith A, Khawli LA. Key factors influencing ADME properties of therapeutic proteins: A need for ADME characterization in drug discovery and development. mAbs. 2016;8(2):229-45.

30. Mulvihill EE, Burke AC, Huff MW. Citrus Flavonoids as Regulators of Lipoprotein Metabolism and Atherosclerosis. Annual review of nutrition. 2016;36:275-99.

\section{Tables}

Due to technical limitations, table 1-3 is only available as a download in the Supplemental Files section.

\section{Figures}


A<smiles>COc1ccc([C@@H]2CC(=O)c3c(O)cc(O)cc3O2)cc1O</smiles>

Figure 1

Chemical structure and pharmacological data of hesperetin

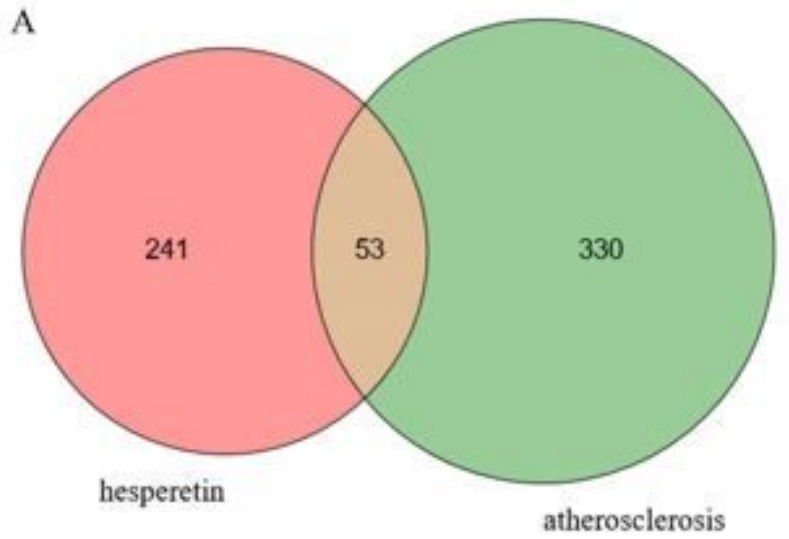

Figure 2

Identification of hesperetin-atherosclerosis crossover targets

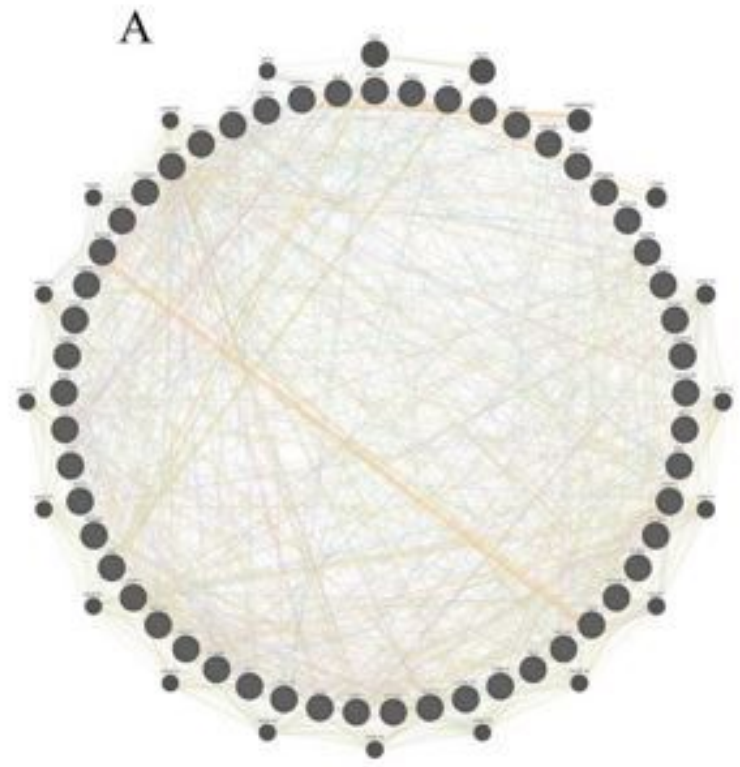

\begin{tabular}{|c|c|c|}
\hline & Co-expression & $49.42 \%$ \\
\hline & Predicted & $17.90 \%$ \\
\hline & Shared protein domains & $13.29 \%$ \\
\hline & Physical Interactions & $10.05 \%$ \\
\hline & Co-localization & $7.04 \%$ \\
\hline & Pathway & $1.95 \%$ \\
\hline & Genetic Interactions & $0.35 \%$ \\
\hline
\end{tabular}

Figure 3 
PPI network of the hesperetin-atherosclerosis crossover targets
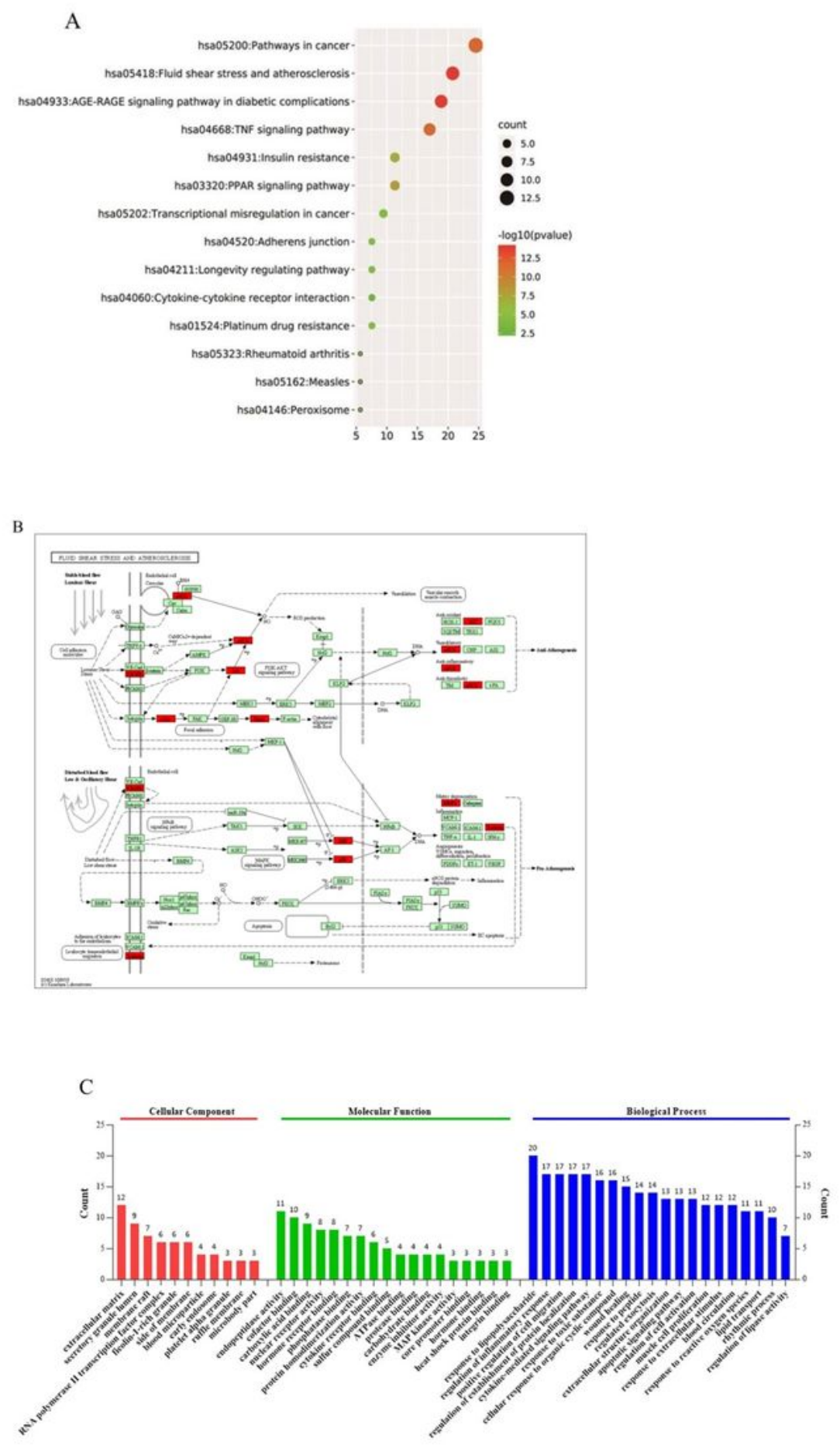

Figure 4

GO and KEGG enrichment analysis of the crossover targets 


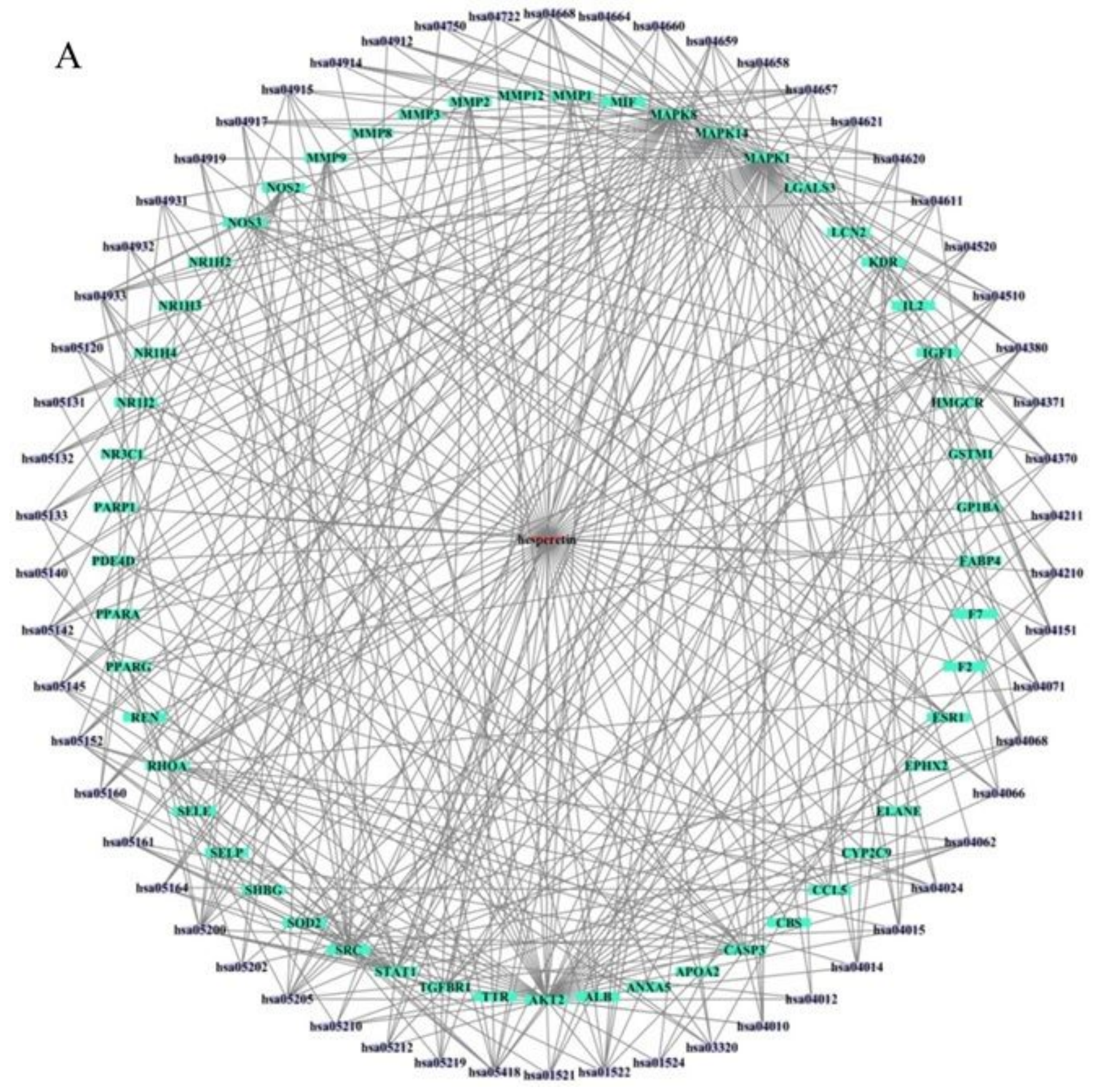

Figure 5

Hesperetin-target-pathway network

\section{Supplementary Files}

This is a list of supplementary files associated with this preprint. Click to download.

- GraphicalAbstract.JPG

- Table1.xlsx

- Table2.xlsx 
- Table3.xIsx

Page 14/14 